\title{
ARTICLE OPEN \\ Quantum teleportation using highly coherent emission from telecom C-band quantum dots
}

\author{
M. Anderson ${ }^{1,2}$, T. Müller (iD ${ }^{1 *}$, J. Huwer ${ }^{1}$, J. Skiba-Szymanska ${ }^{1}$, A. B. Krysa $\mathbb{D}^{3}$, R. M. Stevenson ${ }^{1}$, J. Heffernan ${ }^{4}$, D. A. Ritchie $^{2}{ }^{2}$ and \\ A. J. Shields ${ }^{1}$
}

A practical way to link separate nodes in quantum networks is to send photons over the standard telecom fibre network. This requires sub-Poissonian photon sources in the wavelength band around $1550 \mathrm{~nm}$, with photon coherence times sufficient to enable the many interference-based technologies at the heart of quantum networks. Here, we show that droplet epitaxy InAs/InP quantum dots emitting in the telecom C-band can provide photons with coherence times exceeding 1 ns under low power non-resonant excitation, and demonstrate that these coherence times enable near-optimal interference with a C-band polarisation-encoded laser qubit, with visibilities only limited by the quantum dot multiphoton emission. Using entangled photons, we further show teleportation of such qubits in six different bases with average postselected fidelity reaching $88.3 \pm 4.0 \%$. Beyond direct applications in long-distance quantum communication, the high degree of coherence in these quantum dots is promising for future spin-based telecom quantum network applications.

npj Quantum Information (2020)6:14; https://doi.org/10.1038/s41534-020-0249-5

\section{INTRODUCTION}

Quantum network technologies, ${ }^{1}$ ranging from quantum teleportation ${ }^{2}$ and its applications in quantum communication, ${ }^{3-5}$ to heralded entanglement ${ }^{6}$ and quantum computing, ${ }^{7}$ rely on interference of indistinguishable photons, and demand sources of highly coherent photons with sub-Poissonian statistics. To establish larger-scale quantum networks over the standard telecom fibre network, the photons should further have a wavelength in the minimum loss telecom C-band around $1550 \mathrm{~nm}$.

Solid-state quantum systems, most prominently semiconductor quantum dots (QDs) and defects in diamond, are well-established candidates for emerging quantum technologies. Significant work in this field has developed the basic building blocks of a quantum network, such as near-ideal photon sources, ${ }^{8,9}$ deterministic entanglement between distant spins, ${ }^{10}$ and teleportation of qubits. ${ }^{4}$ However, these systems have in common that their wavelength in the visible and near infrared up to about $900 \mathrm{~nm}$ prevents wider integration using the standard optical fibre infrastructure due to the strong attenuation $(>1 \mathrm{~dB} / \mathrm{km})$ at those wavelengths. Recent efforts have pushed QD emission to the telecom O-band at $1310 \mathrm{~nm}^{5}(0.35 \mathrm{~dB} / \mathrm{km})$, and the $\mathrm{C}$-band $(0.2 \mathrm{~dB} / \mathrm{km})$, where single and entangled photon-pair sources were reported. ${ }^{11-15}$ However, although the coherence times and indistinguishability of C-band QDs under different excitation schemes have recently been studied, ${ }^{16}$ even basic quantum network building blocks such as the interference of photons from separate sources are still outstanding for these technologies.

Alternatively, technologies based on wavelength conversion of single-photon sources or non-linear optical processes have successfully been used to reach the telecom C-band and demonstrate two-photon interference ${ }^{17}$ as well as teleportation over several kilometres, ${ }^{18,19}$ respectively. But these approaches are limited in efficiency due to the losses during the conversion process, or the Poissonian statistics underlying attenuated laser pulses that therefore require an additional layer of complexity to guarantee optimal operation.

Here, we demonstrate coherent emission from InAs/InP QDs emitting in the standard telecom window, through measurement of single and two-photon interference. We show further the power and utility that this photonic platform offers in quantum information by teleporting a telecom C-band polarisation encoded laser qubit.

\section{RESULTS}

Single photon interference

The experiments we report here use metalorganic vapour-phase epitaxy grown droplet InAs QDs on an InP substrate (see Methods for further discussion). A photoluminescence spectrum taken at the pump power where the neutral exciton $(X)$ intensity saturates $\left(P_{\text {sat }}\right)$ is shown in Fig. 1a. We observe several isolated emission lines, and identify the $X$ and biexciton $(X X)$ transitions by polarisation spectroscopy and intensity correlations. Other observed emission lines are most likely resultant from charged excitonic configurations, typically seen in QDs.

The coherence time $T_{2}$ of single photons can then be measured with high temporal resolution through first-order field-correlation measurements using Fourier Transform Spectroscopy. ${ }^{20}$ It depends on the radiative lifetime $T_{1}$ as well as the pure dephasing time $T_{2}^{*}$ via the relation

$\frac{1}{T_{2}}=\frac{1}{2 T_{1}}+\frac{1}{T_{2}^{*}}$

where the radiative lifetime of the QD $X$ transition is measured to be $T_{1}=1.765 \pm 0.013 \mathrm{~ns}$, as shown in Fig. 1b. Two exemplary visibility measurements performed on this transition at excitation powers of $0.75 P_{\text {sat }}$ and $0.03 P_{\text {sat }}$ are given in Fig. $1 c$, with the latter

\footnotetext{
${ }^{1}$ Toshiba Research Europe Limited, 208 Science Park, Milton Road, Cambridge CB4 OGZ, UK. ${ }^{2}$ Cavendish Laboratory, University of Cambridge, JJ Thomson Avenue, Cambridge CB3 OHE, UK. ${ }^{3}$ EPSRC National Epitaxy Facility, University of Sheffield, Sheffield S1 3JD, UK. ${ }^{4}$ Departement of Electronic and Electrical Engineering, University of Sheffield, Sheffield S1 3JD, UK. *email: tina.muller@crl.toshiba.co.uk
} 

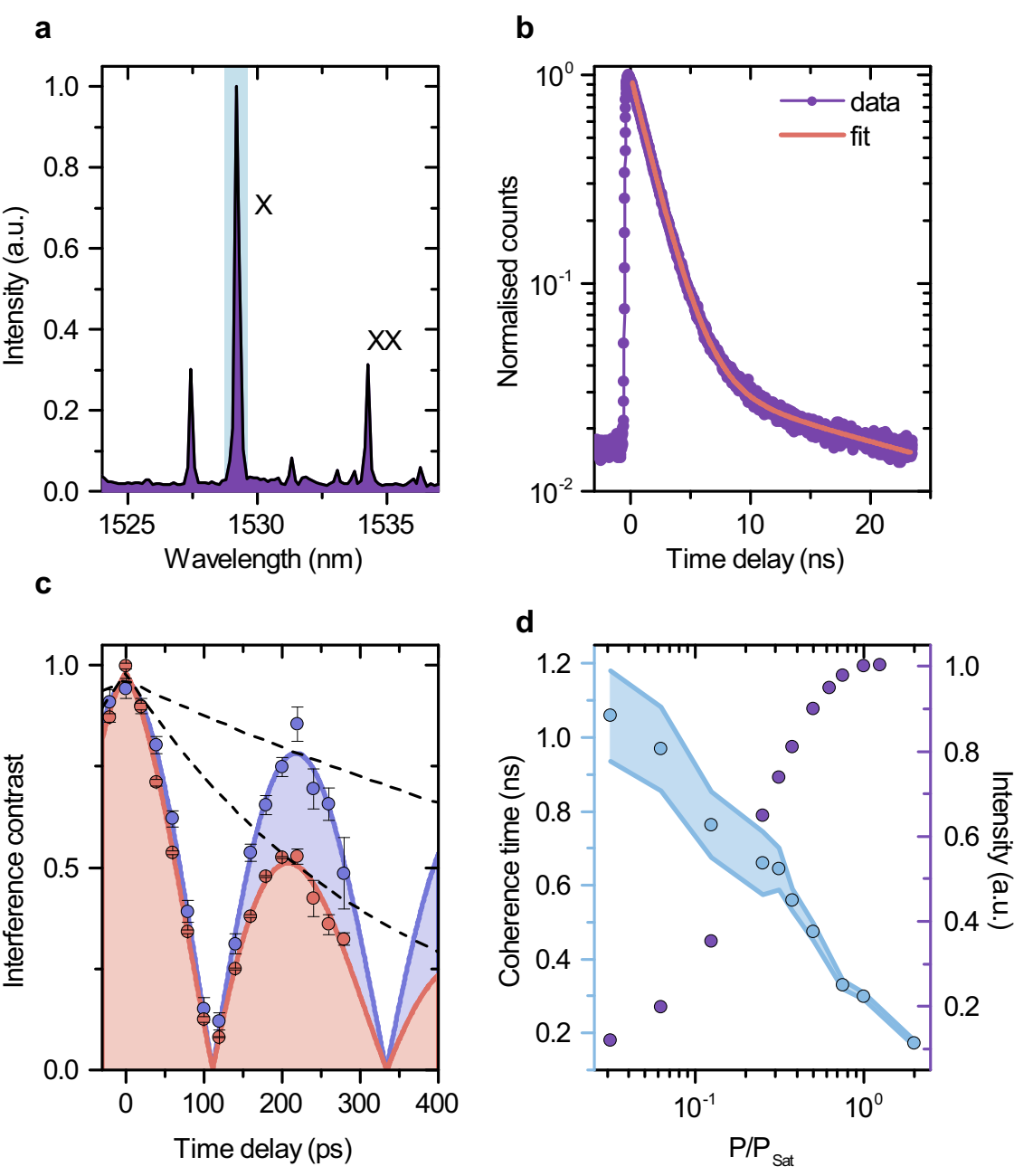

Fig. 1 Single-photon interference. a Spectrum of the QD measured at $P_{\text {sat }}$ with exciton $(X)$ and biexciton $(X X)$ labelled. The shaded area denotes the part of the spectrum used for these measurements. $\mathbf{b}$ Time-resolved intensity of $X$. The solid line is a fit to a biexponential decay. c Single-photon interference measurements of the $X$ at $0.75 P_{\text {sat }}$ (pink) and $0.03 P_{\text {sat }}$ (purple). The visibility oscillates at the beat frequency corresponding to the FSS of the $X$ transition. Solid lines are fits to the data according to a Fourier transform of a double Lorentzian. d Coherence time $\left(T_{2}\right)$ extracted from the fits (blue) and integrated intensity (purple) as a function of excitation power. Error bars on integrated intensity are within the symbol size, and shaded areas denote standard error.

chosen as the lowest power with a sufficient signal-to-noise ratio for the measurement. For both powers, the decay of the visibility displays a characteristic beating pattern in the fringe contrast resulting from the fine-structure splitting (FSS) of the $X$. However, the envelope of the oscillations decays more slowly for lower driving powers. The Fourier transform of a sum of two Lorentzian functions with the same width and different central frequencies was used to fit our data (see Methods for further discussion) from which we extract a FSS of $\Delta E=18.5 \pm 0.2 \mu \mathrm{eV}$ and a coherence time $T_{2}=331 \pm 12 \mathrm{ps}$ at $0.75 P_{\text {sat }}$, where the uncertainty is given by the standard error extracted from a least-squares fitting routine. At the lower pump power, the coherence time remarkably increases to $T_{2}=1058 \pm 134 \mathrm{ps}$, far exceeding the best recently reported values for InAs/InGaAs/GaAs C-band QDs with similar radiative lifetimes. ${ }^{16}$ It even approaches those measured using sub-Kelvin temperatures, ${ }^{21}$ where one expects an almost dephasing free regime.

The power dependence of $T_{2}$ is shown in Fig. 1c, where coherence times are longer and dephasing is reduced for lower pump powers due to a more stable charge environment in the vicinity of the QD under these conditions. ${ }^{22}$ Increasing the excitation power leads to brighter emission from the QD (Fig. 1d) at the cost of increased charge fluctuations and spectral diffusion. We note however that even at maximum $X$ brightness at $P_{\text {sat, }}$ the measured coherence time $\left(T_{2}=299 \pm 9 \mathrm{ps}\right)$ is still sufficient for applications such as a quantum relay. ${ }^{5}$ The fact that our QD maintains a high level of temporal coherence even under saturated pumping conditions where the intensity is the brightest for the $X$ is remarkable, especially with the given excitation scheme where one would typically expect to observe a relatively high level of dephasing. We hypothesise that the measured level of temporal coherence could be a result of the droplet epitaxy growth mode, where the QDs are nominally formed without the presence of a large wetting layer typically seen in Stranski-Krastanov QDs. ${ }^{23}$ The absence, or reduction, of such a wetting layer serves to remove an abundance of charge carriers when exciting non-resonantly. However, a further investigation of the properties of these droplet QDs is required in order to fully determine the origin, as other factors such as the difference in material substrate, strain profile or level of defects may also contribute.

A Fourier transform-limited photon exhibits a coherence time $T_{2}=2 T_{1}$. In this limit, the emitter is fundamentally free from external dephasing processes. For the highest coherence time measured here, $T_{2} / 2 T_{1}=0.298 \pm 0.038$, reducing to $0.085 \pm 0.003$ at the maximum brightness. Notably, the latter is a factor 4 higher 
than recently reported values for $\operatorname{InAs} / \mathrm{InGaAs} / \mathrm{GaAs}$ C-band QDs under non-resonant excitation at similar relative excitation power, ${ }^{16}$ translating to a reduction of the pure dephasing rate $1 / T_{2}^{*}$ for the InAs/InP system used here. Only under strict resonant s-shell excitation, which is less disruptive than the non-resonant excitation scheme used here, has this value been shown to approach the Fourier limit. ${ }^{9,24}$ Given the current weak confinement of the QD light in the planar cavity with no appreciable Purcell enhancement, ${ }^{25}$ there is potential to reduce radiative lifetime $T_{1}$ further by enhancing the coupling to the cavity mode, bringing the emission even closer to the transform limit. ${ }^{26}$

Two-photon interference with a laser

The most fundamental quantum network technology enabled by the long coherence times is the interference of two photons. To demonstrate this, we interfere a QD photon with a telecomwavelength laser qubit of the form

$\left|L_{\text {in }}\right\rangle=\cos \left(\frac{\theta}{2}\right)\left|H_{L}\right\rangle+e^{i \varphi} \sin \left(\frac{\theta}{2}\right)\left|V_{L}\right\rangle$,

where $\theta$ and $\varphi$ parameterise the polarisation state on the Poincaré sphere. This is the first step in the quantum teleportation protocol presented below, and so we operate this experiment with a QD that is suitable not only for the interference, but the teleportation also. In addition to the long temporal coherence time, a QD with low FSS, leading to high entanglement fidelity, and also a high photon count rate to collect the necessary correlation statistics is required. We typically find coherence times exceeding $150 \mathrm{ps}$ in $80 \%$ of QDs in our sample when driving at $P_{\text {sat }}$ to extract maximum count rates. Therefore, the requirement of a $\mathrm{QD}$ with sufficient FSS becomes the important parameter to optimise. For the QD used in the remainder of the paper, we measured an FSS of $5.7 \pm 0.2 \mu \mathrm{eV}$, a maximum entanglement fidelity of $91.0 \pm 0.5 \%$ and saturation count rates typically around $300 \mathrm{k}$ counts/s for $X$.

We start by measuring the QD autocorrelation $g_{O D}^{(2)}(\tau)$, to confirm the single-photon nature of the emission. The autocorrelation of the QD at saturation power is shown in Fig. 2a, where strong bunching can be seen for short delays in addition to the antibunching dip at the zero time delay. This bunching is due to a long-lived shelving state such as a dark state accessible by a spin flip, which we capture by adding a third level to the simple two-level system often used to model $X$ emission. We further find that the shape of the $g^{(2)}(\tau)$ dip is best described by adding a fourth state ${ }^{27}$ capturing fast feeding processes to the $X$, which leads to the fitting function

$g_{Q D}^{(2)}(\tau)=1-(1+A+B) e^{-|\tau| / \tau_{1}}+A e^{-|\tau| / \tau_{2}}+B e^{-|\tau| / \tau_{3}}$,

where $A$ and $B$ denote the coupling strength from the additional levels, $\tau_{1}$ is a timescale that depends on the radiative lifetime of the $X$ as well as the pumping rate, and $\tau_{2}, \tau_{3}$ are the timescales of the additional decay processes which populate the $X$. Note that for lower powers, $B \rightarrow 0$ and the behaviour reduces to a standard three-level model. To quantify the influence of uncorrelated background events which causes the degree of second-order

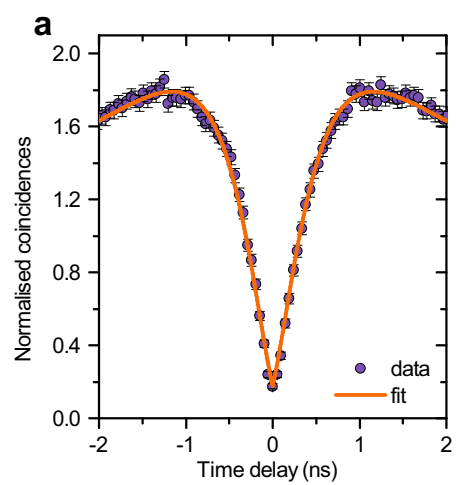

C

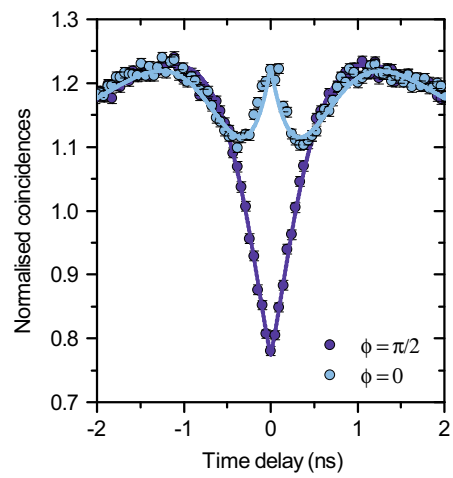

b

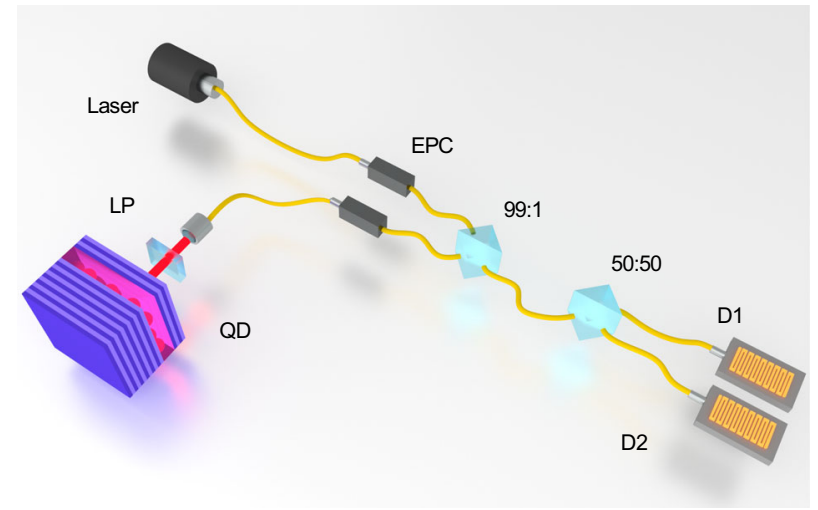

d

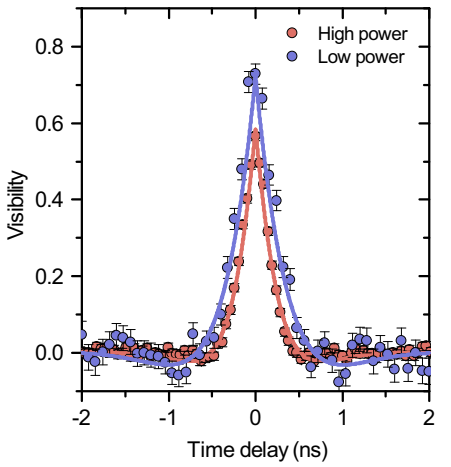

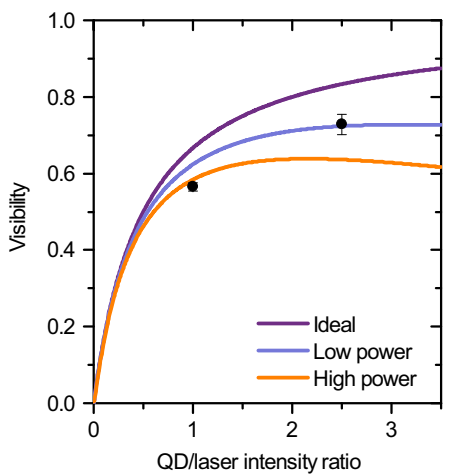

Fig. 2 Two-photon interference of a laser qubit. a Autocorrelation of the QD fitted to a multi-level model (solid line). b Experimental schematic. A laser photon is interfered with a QD photon on a 99:1 unbalanced beamsplitter. Correlation measurements are performed on photons which exit via the same port of the beamsplitter using a standard Hanbury-Brown and Twiss (HBT) setup with a 50:50 beamsplitter and superconducting nanowire single-photon detectors (D1 \& D2). The photon polarisation is controlled using a linear polariser (LP) and electronic polarisation controllers (EPCs). c Correlation measurements of TPI for indistinguishable (blue) and distinguishable (purple) photons. d Corresponding visibility of the TPI for the high and low-intensity regimes discussed in the text. e Calculated TPI visibility as a function of the relative $\mathrm{QD} /$ laser intensity ratio $\eta / a^{2}$ for the ideal case where the degree of second-order coherence $g_{H B T}^{(2)}(0)=0$, and for the $g_{H B T}^{(2)}(0)=0.177$ and 0.095 corresponding to recorded values at $P_{\text {sat }}$ and at $P_{\text {sat }} / 20$, respectively. Black points denote raw experimental values extracted from d. There is no correction for detection response. 
coherence $g^{(2)}(0)$ to deviate from the ideal value of 0 , we define the function

$g_{H B T}^{(2)}(\tau)=\frac{g_{Q D}^{(2)}(\tau)+2 \beta+\beta^{2}}{(1+\beta)^{2}}$,

where $\beta$ is the contribution of the uncorrelated background intensity. This model is convolved with a Gaussian function with a 90 ps FWHM to account for the measured detector response, and is used to fit the autocorrelation data in Fig. 2a. From the fit we find $g^{(2)}(0)=0.177$ with a fitted $\beta=0.10 \pm 0.02$ at $P_{\text {sat }}$. This moderate contribution of background is due to uncorrelated light emission from the semiconductor environment of the QD, spectrally close to the quantum dot, and is power dependent. A similar measurement at $P_{\text {sat }} / 20$ which reveals a $g^{(2)}(0)=0.095$ with a fitted $\beta=0.05 \pm 0.01$. We further determine the values $A=$ $1.97 \pm 0.08, B=1.96 \pm 1.33, \tau_{1}=0.36 \pm 0.03 \mathrm{~ns}, \tau_{2}=2.18 \pm 0.03 \mathrm{~ns}$ and $\tau_{3}=0.19 \pm 0.05 \mathrm{~ns}$ at $P_{\text {sat }}$ and $A=0.90 \pm 0.01, \tau_{1}=1.53 \pm$ $0.02 \mathrm{~ns}, \tau_{2}=40.80 \pm 0.20 \mathrm{~ns}$ at $P_{\text {sat }} / 20$. The high error on the coupling $B$ suggests that the effect is very weak, thus confirming that as the power is lowered, the standard 3-level model is recovered.

A schematic of the two-photon interference (TPI) measurement is shown in Fig. 2b. Photons are overlapped at a 99:1 beamsplitter, after which correlation measurements are performed. If the laser and dot photons are distinguishable (due to their orthogonal polarisation), we expect a dip at zero delay due to the singlephoton nature of the photon from the dot. If however the input photons are indistinguishable and identical in all degrees of freedom (in the co-polarised case), we expect to see bunching at zero delay due to two-photon interference superimposed on the dip. $^{28}$ This can be described by a modified version of the twophoton correlation function for dissimilar photon sources presented by Bennett et al., ${ }^{29}$ taking into account differences in our experimental setup and background contributions. The form of the correlation function is then

$g^{(2)}(\tau)=1+\frac{2 \eta a^{2}\left(e^{-|\tau| / T_{2}} \cos \left(\Delta E_{L} \tau / \hbar\right) \cos ^{2} \phi\right)+\eta^{2}\left(g_{Q D}^{(2)}(\tau)-1\right)}{\left(\eta+a^{2}+\beta\right)^{2}}$,

where $\eta$ is the dot intensity, $a^{2}$ is the laser intensity, $\beta$ is the uncorrelated background contribution, $\Delta E_{L}$ is the detuning between QD and laser photon, and $\phi$ gives the polarisation difference between the two photons $(\phi=0$ and $\phi=\pi / 2$ for coand cross-polarised photons, respectively). The interference visibility can be calculated from the contrast of the co- and cross-polarised correlation measurements as $V(\tau)=g_{\|}^{(2)}(\tau) / g_{\perp}^{(2)}(\tau)-1$, where $g_{\|}^{(2)}(\tau)$ and $g_{\perp}^{(2)}(\tau)$ are the correlation functions for the co- and cross-polarised cases, respectively.

We perform TPI for realistic teleportation conditions, where the QD is excited at $P_{\text {sat }}$ and $\eta / a^{2}=1$, as well as for more ideal conditions, where the driving power is reduced to $P_{\text {sat }} / 20$, and $\eta / a^{2}=2.5$. As an example, the resulting correlation measurements for the higher power parameters are shown in Fig. 2c. The calculated visibility for both sets of parameters can be seen in Fig. $2 \mathrm{~d}$, where the peak raw (fitted) values of $56.5 \pm 1.1 \%(58.6 \pm 0.5 \%)$ and $72.8 \pm 2.7 \%(72.4 \pm 0.7 \%)$ are obtained. A small energy detuning of $\sim 2 \mu \mathrm{eV}$ (see Methods for further details) between the laser and the QD photons accounts for the small beating in the measured visibility. From the fits we can further determine $\tau_{c}=$ $294 \pm 9 \mathrm{ps}$ at $P_{\text {sat }}$ and $\tau_{c}=471 \pm 29 \mathrm{ps}$ at $P_{\text {sat }} / 20$. The coherence time at $P_{\text {sat }}$ is in agreement with the value measured using the Ml for this QD (262 \pm 19 ps, data not shown), and is also comparable to the value shown by the QD presented in Fig. 1, both in terms of raw coherence time and the ratio $T_{2} / 2 T_{1}\left(T_{1}=1.580 \pm 0.006 \mathrm{~ns}\right)$ at this power. We find that this coherence time is also consistent with a measurement of the mutual degree of indistinguishability for the QD presented in Fig. 1, which can be evaluated using a standard Hong-Ou-Mandel (HOM) interferometer. ${ }^{28}$ Here, we determine a peak raw HOM visibility ${ }^{30}$ of $V_{\text {HOM,raw }}=62.9 \pm 3.6 \%$ and a coherence time of $303 \pm 20$ ps when exciting at $P_{\text {sat }}$ with a delay between photons of $6.25 \mathrm{~ns}$.

In practice, both the degree of second-order coherence of the QD $\left(g^{(2)}(0)\right)$ and the QD/laser intensity ratio $\eta / a^{2}$ limit the maximum achievable visibility. In our case, for both power settings, the visibility is above $85 \%$ of the ideal value where $g^{(2)}(0)=0$, and is well described when taking the degree of second-order coherence at the respective powers into account, as shown in Fig. 2e.

\section{Quantum teleportation}

One direct application of this type of interference is the teleportation of a qubit, a fundamental operation in quantum information technologies. The longer the temporal coherence of the interfering photons, the more photons are teleported with high fidelity. We choose to teleport a telecom-wavelength polarisation-encoded laser qubit, as this is the most relevant for the practical application of using a quantum relay to extend the reach of existing quantum key distribution systems.

The quantum teleportation protocol relies on the distribution of an entangled pair of photons between an intermediate station, Charlie, and Bob. A Bell state measurement is then performed on the input qubit from Alice and one of the entangled photons (Charlie). This measurement projects the wavefunction of Bob's photon into the state of the input, up to a unitary transform. ${ }^{31}$ In this experiment, we use the entanglement generated by the biexciton radiative cascade, which results in the two-photon entangled state

$$
|\Psi(\tau)\rangle=\frac{1}{\sqrt{2}}\left[\left|H_{X X} H_{X}\right\rangle+e^{i \Delta E \tau / \hbar}\left|V_{X X} V_{X}\right\rangle\right]
$$

where $H$ and $V$ denote the horizontal and vertical polarisation eigenbasis of the QD. The teleportation protocol is illustrated in the sketch of our experimental implementation in Fig. 3a. While similar setups have been successfully used to teleport photons at shorter wavelengths, ${ }^{4,5}$ no QD sources have so far been available to conduct this experiment in the technologically important wavelength region around $1550 \mathrm{~nm}$. Here, the $X$ photon is overlapped with the input laser qubit (Alice) at the 99:1 beamsplitter, with the intensity of the laser set to match the intensity of the QD to optimise both TPI performance and threephoton coincidence rates. We then perform a Bell-state measurement using a polarising beamsplitter (PBS) and detectors $D_{H}$ and $D_{V}$ calibrated to the QD eigenbasis (Charlie). The $X X$ photon is finally analysed at a PBS by aligning the detectors $D_{P}$ and $D_{Q}$ to the expected output state basis (Bob). Experimentally, coincidence detection of photons are measured at Charlie and Bob in order to generate third-order correlations maps as a function of the time delays $\tau_{C}=t_{D_{V}}-t_{D_{H}}$ and $\tau_{B}=t_{D_{P(Q)}}-t_{D_{H}}$. A successful Bell-state measurement heralds the teleportation $\left(\tau_{C}=0\right)$, which can then be correlated with the photon detections at Bob. The teleportation fidelity is then calculated as ${ }^{4}$

$f_{P}^{T}=\frac{g_{P}^{(3)}\left(\tau_{C}, \tau_{B}\right)}{g_{P}^{(3)}\left(\tau_{C}, \tau_{B}\right)+g_{Q}^{(3)}\left(\tau_{C}, \tau_{B}\right)}$,

where $g_{P(Q)}^{(3)}\left(\tau_{C}, \tau_{B}\right)$ is the third-order correlation function for Bob's photon detected in polarisation $P(Q)$, and serves as a measure of the expected (unexpected) output polarisation compared to the input. By sending horizontal $(H)$, vertical $(V)$, diagonal $(D=(H+V) / \sqrt{2})$, antidiagonal $(A=(H-V) / \sqrt{2})$, right-hand circular $(R=(H-i V) / \sqrt{2})$ and left-hand circular $(L=(H+i V) / \sqrt{2})$ weak coherent laser input qubits, corresponding to the six symmetrically distributed polarisation states on the 
a
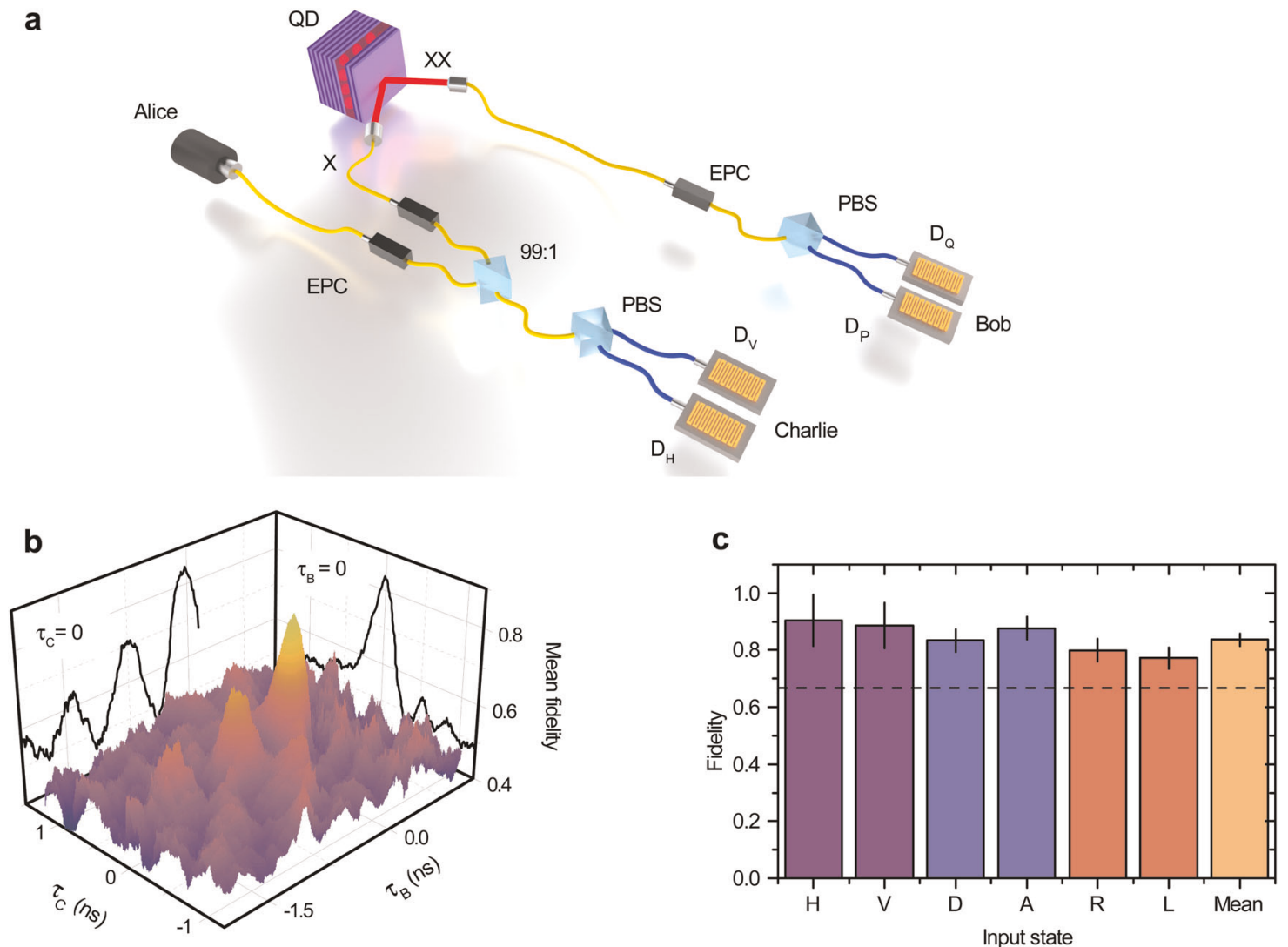

Fig. 3 Quantum teleportation of a laser qubit. a Experimental schematic. An input polarisation encoded laser qubit is interfered with an $X$ photon on a 99:1 beamsplitter. At Charlie, a Bell state measurement is performed using a polarising beamsplitter (PBS) and superconducting single-photon detectors $\left(D_{H} \& D_{V}\right)$. Bob then uses polarisation resolved correlations to analyse the output state in different polarisation bases at detectors $\left(D_{P} \& D_{Q}\right)$. b Mean fidelity coincidence map for the most significant post-selection window size of 203 ps. The peak fidelity at $\tau_{C}=$ $\tau_{B}=0$ is $83.6 \pm 2.2 \%$. c Individual fidelities for the six input polarisation states and the corresponding mean fidelity. The fidelity exceeds the classical limit of $2 / 3$ (dotted line) for each basis in the six-state teleportation protocol.

Poincaré sphere, we can measure correlations in three different polarisation bases at Bob to determine the mean fidelity of our teleporter.

For the dot presented in Fig. 2, driven at $P_{\text {sat }}$, the resulting mean fidelity coincidence map for the post-selection window corresponding to the most statistically significant teleportation is displayed in Fig. 3b, where the point of highest fidelity is centred on $\tau_{C}=\tau_{B}=0$. This corresponds to the three-photon coincidence where the input polarisation state is mapped to the output photon at Bob, and a maximum mean fidelity of $83.6 \pm 2.2 \%$ for an equivalent window size of 203 ps is achieved. This fidelity exceeds the classical threshold of $2 / 3$ by 7.8 standard deviations, and is also 5.1 standard deviations above the limit imposed for secure implementations of six-state secret key sharing protocols. ${ }^{32}$ Other relevant features can be examined by taking a cut along the $\tau_{C}=0$ axis, as shown in Fig. 3b, where we see oscillations in the fidelity resulting from the time-evolving nature of the two-photon entangled source. Indeed, the beat frequency is given exactly by the FSS of the QD. A cut along the $\tau_{B}=0$ axis shows the highfidelity teleportation heralding peak with width set by the $X$ coherence time. It is clear from this cut-through that a small amount of residual detuning of the laser from the QD is present, accounting for difference in the peak width from Fig. $2 \mathrm{~d}$ in the TPI measurement. This is most likely as a result the $5.7 \mu \mathrm{eV}$ splitting combined with the $\sim 2 \mu \mathrm{eV}$ accuracy of the overlapping routine.

We use temporal post-selection to filter out the successfully teleported photons. Our teleporter is limited temporally along $\tau_{C}$ by the interference visibility of the laser and QD photon, with a timescale given by the $X$ coherence time and the detector resolution. A longer $X$ coherence time will therefore directly lead to a relaxation of post-selection conditions and therefore more efficient teleportation. Along $\tau_{B}$, the evolution of the quantum state due to the FSS, together with the detector resolution, limits the maximum window size. When varying the window size, smaller windows lead to higher fidelities at the cost of teleporting fewer photons within that window. For larger window sizes, more photons are teleported, but the signal starts to be washed out and the teleportation fidelity decreases.

Varying the temporal post-selection window size, we find the mean fidelity increases to a maximum of $88.4 \pm 4.0 \%$ for an equivalent post-selection window size of $103 \mathrm{ps,} \mathrm{which} \mathrm{is}$ 5.4 standard deviations above the classical threshold. The main factors that contribute to this maximum measured teleportation fidelity are the TPI visibility and the entanglement fidelity. For the relatively high excitation power used here, the non-zero $g_{H B T}^{2}(0)$ value of the $X$ limits the achievable TPI visibility, as shown in Fig. 2e. Consequently, this lowers the expected teleportation fidelity as fewer photons are interfering and taking the correct path at Charlie, leading to a higher proportion of incorrectly heralded photons measured at Bob. Further, the finite $g_{H B T}^{2}(0)$ value also limits the entanglement fidelity, as it leads to photon pairs with uncorrelated polarisation. For low excitation power on the other hand, the reduced brightness and non-zero detector dark counts lead to significantly fewer three-photon coincidences and reduced fidelity. The exact relative contribution of these factors with respect to the teleportation fidelity is outside the scope of this investigation, however, we note it is generally seen that the TPI visibility has a stronger impact on the teleportation fidelity. ${ }^{33}$ 
Individual basis fidelities from Fig. 3b are displayed in Fig. 3c, where we note each one is above both the classical and six-state protocol thresholds. As such, our teleporter offers a robust and universal platform where teleportation of any arbitrary input state can be realised with high fidelity.

\section{DISCUSSION}

We have demonstrated the highly coherent emission from a telecom C-band quantum light source based on semiconductor QDs by measuring a single-photon coherence time exceeding $1 \mathrm{~ns}$ and a $T_{2} / 2 T_{1}$ ratio of 0.298 at low $C W$ excitation powers. This compares well with state-of-the-art systems even when we use non-optimal, but highly practical, non-resonant excitation schemes. We have found that the emission remains sufficiently coherent even when driven at saturation power to enable interference measurements with a weak coherent source. Taking into account the limitations imposed by the degree of secondorder coherence of the QD, we obtain near-perfect visibility. Device performance could be further improved by the addition of a solid immersion lens, which would increase both the excitation and collection efficiency, resulting in higher detected count rates, ${ }^{33,34}$ and enabling the benefit of the higher coherence under lower excitation powers.

We further showed how these experiments can be combined to form basic building blocks of a quantum network, by measuring proof-of-principle quantum teleportation of telecom C-band qubits. We note that these results are achieved in a system with significant differences in materials, QD growth, device structure and operation compared to standard GaAs-based QDs, and demonstrate for the first time the combination of all necessary properties for the operation of a quantum relay in such a device. The teleportation shown here with emission wavelength in the telecom C-band straightforwardly enables extension of the reach of existing QKD systems, using simple non-resonant driving and relying only on standard industry growth and fabrication techniques in a material compatible with on-chip integrated detection. In addition, on-demand operation of the relay described here, which is essential for real-world applications, could straightforwardly be achieved using pulsed optical excitation.

The low degree of spectral wandering and stable transition energy revealed by the long coherence times could further facilitate resonant excitation schemes, where we expect the true limits of coherence in emitted photons and the underlying spin states to be revealed. For example, it has been demonstrated that resonant two-photon excitation can lead to photon emission closer to the transform limit, ${ }^{34}$ while still maintaining the entanglement properties necessary for quantum teleportation. ${ }^{33}$ Such a scheme could make our system available for a whole range of applications pioneered using short-wavelength solid-state systems, at a wavelength that can be directly integrated with long-distance quantum networks.

\section{METHODS}

\section{Sample design and measurement setup}

Our device consists of metalorganic vapour-phase epitaxy grown droplet InAs QDs on an InP substrate embedded in a planar cavity between pdoped repeated layers of distributed Bragg reflectors on the top and ndoped layers on the bottom. The sample is similar to that used in our recent work. ${ }^{15}$ The device is operated at $8.5 \mathrm{~K}$ in a temperature-controlled Helium vapour cryostat with an electrically open configuration, as we found application of a gate voltage had little effect on improving the measured coherence times for this device. Emission from a single QD is collected using a confocal microscope $(\mathrm{NA}=0.68)$ coupled to a standard telecom single-mode fibre. The light is then guided to either a grating spectrometer and detected on an $\operatorname{lnGaAs}$ array, or to a free-space transmission grating (resolution $\sim 0.5 \mathrm{~nm}$ ) to spatially separate the $X$ and
$X X$ emission lines. After separation, the light is fibre coupled and guided to either the TPI or teleporation setup (described in the main text), before being detected on SNSPDs with a measured pair response of around $90 \mathrm{ps}$ FWHM. For the coherence time measurements, non-resonant CW excitation at $785 \mathrm{~nm}$ is used to excite the dot. For the TPI and teleportation measurements, the non-resonant CW excitation wavelength used is $1310 \mathrm{~nm}$, as this increases the count rates from the neutral exciton and biexciton transitions. We found that the coherence times are not affected by the wavelength change, which only serves to increase the emission count rate for the two- and three-photon measurements. The reference frame for polarisation labelling is given by the QD FSS. The polarisation of highest $X$ energy is defined to be $V$, from which all other polarisations can be deduced. For the EPC calibration which defines the detection system to the various polarisation states, a laser reference is used to simulate the emission of the QD, determined using a linear polariser, half-wave plate and quarter-wave plate. One of the outputs of the beamsplitters at Charlie and Bob is then minimised for the different detection bases. The same minimisation is then performed for the input laser, matching the input polarisation reference frame to that of the QD. The calibration is carried out similarly for the TPI. The spectral overlap of the laser and the QD is achieved using a grating spectrometer and a fitting routine which minimises the spectral energy difference with a resolution of $\sim 2 \mu \mathrm{eV}$.

\section{Michelson interferometer}

We use a single-mode optical fibre-based Michelson interferometer, where an optical delay line allows us to vary the delay between the arms coarsely and a fibre stretcher to measure interference fringes. When one arm of the interferometer is varied using the fibre stretcher, we measure singlephoton interference as oscillations in the output intensity. Along with the delay line and fibre stretcher, Faraday mirrors are used at the end of both arms to compensate for polarisation rotations and fluctuations in the fibres. The interference fringe visibility is defined as

$V=\frac{I_{\max }-I_{\min }}{I_{\max }+I_{\min }}$

where $I_{\max }$ and $I_{\min }$ correspond to the cases of constructive and destructive interference, respectively. Interference fringes are then fitted to extract this visibility at different coarse delays. We measure the interference pattern of a broadband light source to determine the zero delay setting, and use the visibility measured from a narrowband laser to calibrate the efficiency of our setup and normalise the data. Interference fringes are fitted to extract the visibility. The Fourier transform of a sum of two Lorentzian functions with the same width and different central frequencies is then used to fit our data, and has the form

$A_{0} \exp \left(-|\Delta \tau| / T_{2}\right) \cos (\Delta E \Delta \tau / \hbar)$

where $A_{0}$ is the fringe contrast at zero delay, $T_{2}$ is the coherence time, and $\Delta E$ is the central energy difference. The fringe contrast $A_{0}$ accounts for experimental imperfections, but remains close to unity for all measurements presented here. Note that looking at both components of the split $X$ transition instead of filtering out one of them using a linear polariser provides an accurate, independent measurement of the FSS extracted from the beating period, and further gives access to extra information such as the relative intensity or coherence time of the two transitions.

\section{Error analysis}

For fitted parameters and values, quoted errors are one standard deviation resulting from a standard least-squares fitting routine. For correlation data, the main source of errors in these experiments is the Poissonian counting statistics. Quoted error bars on correlation data denote this.

\section{Reporting summary}

Further information on research design is available in the Nature Research Reporting Summary linked to this article.

\section{DATA AVAILABILITY}

The data that support the findings of this study are available from the corresponding author upon reasonable request. 


\section{CODE AVAILABILITY}

The scripts used to analyse the data collected in this study are available from the corresponding author upon reasonable request.

Received: 21 August 2019; Accepted: 9 January 2020;

Published online: 31 January 2020

\section{REFERENCES}

1. Kimble, H. J. The quantum internet. Nature 453, 1023-1030 (2008).

2. Fattal, D., Diamanti, E., Inoue, K. \& Yamamoto, Y. Quantum teleportation with a quantum dot single photon source. Phys. Rev. Lett. 92, 037904 (2004).

3. Briegel, H.-J., Dür, W., Cirac, J. I. \& Zoller, P. Quantum repeaters: the role of imperfect local operations in quantum communication. Phys. Rev. Lett. 81, 5932-5935 (1998).

4. Nilsson, J. et al. Quantum teleportation using a light-emitting diode. Nat. Photonics 7, 311-315 (2013).

5. Huwer, J. et al. Quantum-dot-based telecommunication-wavelength quantum relay. Phys. Rev. Appl. 8, 024007 (2017).

6. Bernien, $\mathrm{H}$. et al. Heralded entanglement between solid-state qubits separated by three metres. Nature 497, 86-90 (2013).

7. Knill, E., Laflamme, R. \& Milburn, G. J. A scheme for efficient quantum computation with linear optics. Nature 409, 46-52 (2001).

8. Somaschi, N. et al. Near-optimal single-photon sources in the solid state. Nat Photonics 10, 340-345 (2016).

9. Ding, $X$. et al. On-demand single photons with high extraction efficiency and near-unity indistinguishability from a resonantly driven quantum dot in a micropillar. Phys. Rev. Lett. 116, 020401 (2016).

10. Humphreys, P. C. et al. Deterministic delivery of remote entanglement on a quantum network. Nature 558, 268-273 (2018).

11. Kim, D. et al. Photoluminescence of single, site-selected, InAs/InP quantum dots in high magnetic fields. Appl. Phys. Lett. 87, 212105 (2005).

12. Zeuner, K. D. et al. A stable wavelength-tunable triggered source of single photons and cascaded photon pairs at the telecom c-band. Appl. Phys. Lett. 112 173102 (2018)

13. Carmesin, C. et al. Structural and optical properties of $\ln A s /(\operatorname{In}) G a A s / G a A s$ quantum dots with single-photon emission in the telecom c-band up to $77 \mathrm{k}$. Phys. Rev. B 98, 125407 (2018).

14. Olbrich, F. et al. Polarization-entangled photons from an InGaAs-based quantum dot emitting in the telecom C-band. Appl. Phys. Lett. 111, 133106 (2017).

15. Müller, T. et al. A quantum light-emitting diode for the standard telecom window around 1550 nm. Nat. Commun. 9, 862 (2018).

16. Nawrath, $C$. et al. Coherence and indistinguishability of highly pure single photons from non-resonantly and resonantly excited telecom C-band quantum dots. Appl. Phys. Lett. 115, 023103 (2019).

17. Weber, J. H. et al. Two-photon interference in the telecom C-band after frequency conversion of photons from remote quantum emitters. Nat. Nanotechnol. 14, 23-26 (2019).

18. Sun, Q.-C. et al. Quantum teleportation with independent sources and prior entanglement distribution over a network. Nat. Photonics 10, 671-675 (2016).

19. Valivarthi, R. et al. Quantum teleportation across a metropolitan fibre network. Nat. Photonics 10, 676-680 (2016).

20. Zwiller, V., Aichele, T. \& Benson, O. Single-photon fourier spectroscopy of excitons and biexcitons in single quantum dots. Phys. Rev. B 69, 165307 (2004).

21. Reimer, M. E. et al. Overcoming power broadening of the quantum dot emission in a pure wurtzite nanowire. Phys. Rev. B 93, 195316 (2016).

22. Berthelot, A. et al. Unconventional motional narrowing in the optical spectrum of a semiconductor quantum dot. Nat. Phys. 2, 759-764 (2006).

23. Skiba-Szymanska, J. et al. Universal growth scheme for quantum dots with low fine-structure splitting at various emission wavelengths. Phys. Rev. Appl. 8, 014013 (2017).

24. Moody, G. et al. Electronic enhancement of the exciton coherence time in charged quantum dots. Phys. Rev. Lett. 116, 037402 (2016).

25. Gérard, J. M. et al. Enhanced spontaneous emission by quantum boxes in a monolithic optical microcavity. Phys. Rev. Lett. 81, 1110-1113 (1998).

26. Bennett, A. J. et al. Cavity-enhanced coherent light scattering from a quantum dot. Sci. Adv. 2, e1501256 (2016).
27. Kitson, S. C., Jonsson, P., Rarity, J. G. \& Tapster, P. R. Intensity fluctuation spectroscopy of small numbers of dye molecules in a microcavity. Phys. Rev. A $\mathbf{5 8}$ 620-627 (1998).

28. Hong, C. K., Ou, Z. Y. \& Mandel, L. Measurement of subpicosecond time intervals between two photons by interference. Phys. Rev. Lett. 59, 2044-2046 (1987).

29. Bennett, A. J., Patel, R. B., Nicoll, C. A., Ritchie, D. A. \& Shields, A. J. Interference of dissimilar photon sources. Nat. Phys. 5, 715-717 (2009).

30. Patel, R. B. et al. Postselective two-photon interference from a continuous nonclassical stream of photons emitted by a quantum dot. Phys. Rev. Lett. 100, 207405 (2008).

31. Jacobs, B. C., Pittman, T. B. \& Franson, J. D. Quantum relays and noise suppression using linear optics. Phys. Rev. A 66, 052307 (2002).

32. Chau, H. F. Practical scheme to share a secret key through a quantum channel with a $27.6 \%$ bit error rate. Phys. Rev. A 66, 060302 (2002).

33. Reindl, M. et al. All-photonic quantum teleportation using on-demand solid-state quantum emitters. Sci. Adv. 4, eaau1255 (2018).

34. Huber, D. et al. Highly indistinguishable and strongly entangled photons from symmetric GaAs quantum dots. Nat. Commun. 8, 15506 (2017).

\section{ACKNOWLEDGEMENTS}

The authors acknowledge partial financial support from the Engineering and Physical Sciences Research Council and the UK's innovation agency, Innovate UK. M.A. gratefully acknowledges support from the Industrial CASE award funded by the EPSRC and Toshiba Research Europe Limited.

\section{AUTHOR CONTRIBUTIONS}

A.J.S, R.M.S, D.A.R. and J.He. conceived the experiment and supervised the project. J. S.-S., T.M., J.H., A.B.K. and R.M.S. designed the QD structures. A.B.K. engineered the epitaxial process, grew the diode wafers and performed basic characterisation of the epitaxial material. J.S.-S. coordinated growth and manufactured the device. M.A. and T.M. took the measurements and analysed the data with support from J.H. M.A., T.M. R.M.S. and A.J.S. wrote the manuscript. All authors discussed the results and commented on the manuscript.

\section{COMPETING INTERESTS}

The authors declare no competing interests.

\section{ADDITIONAL INFORMATION}

Supplementary information is available for this paper at https://doi.org/10.1038/ s41534-020-0249-5.

Correspondence and requests for materials should be addressed to T.M.

Reprints and permission information is available at http://www.nature.com/ reprints

Publisher's note Springer Nature remains neutral with regard to jurisdictional claims in published maps and institutional affiliations.

Open Access This article is licensed under a Creative Commons Attribution 4.0 International License, which permits use, sharing, adaptation, distribution and reproduction in any medium or format, as long as you give appropriate credit to the original author(s) and the source, provide a link to the Creative Commons license, and indicate if changes were made. The images or other third party material in this article are included in the article's Creative Commons license, unless indicated otherwise in a credit line to the material. If material is not included in the article's Creative Commons license and your intended use is not permitted by statutory regulation or exceeds the permitted use, you will need to obtain permission directly from the copyright holder. To view a copy of this license, visit http://creativecommons. org/licenses/by/4.0/.

(c) The Author(s) 2020 